\title{
La infección por el virus del papiloma humano, un posible marcador biológico de comportamiento sexual en estudiantes universitarios
}

Miguel A Sánchez-A lemán, Q FB, M en $C_{\text {, }}^{(1)}$ Felipe U ribe-Salas, MC, MSP, M en $C$, ${ }^{(1)}$ $C$ arlos J Conde-González, Q BP, M en C, D r en C.(1)

\begin{abstract}
Sánchez-Alemán MA, Uribe-Salas F, Conde-González CJ. La infección por el virus del papiloma humano, un posible marcador biológico de comportamiento sexual en estudiantes universitarios. Salud Publica Mex 2002;44:442-447. El texto completo en inglés de este artículo está disponible en: http://www.insp.mx/salud/index.html

\section{Resumen}

Objetivo. Estimar la prevalencia de infección por el virus del papilo ma humano (VPH) en estudiantes universitarios y utilizar dicha frecuencia como un marcador biológico para evaluar el comportamiento sexual. Material y métodos Se realizó un estudio transversal, en estudiantes de la Universidad Autóno ma del estado de Morelos, México, durante el periodo 2000-2001. Se aplicó un cuestionario y se colectaron muestras genitales para detectar ADN de losVPH oncogénicos. Los datos se analizaron utilizando pruebas de Ji cuadrada y razones de momios. Resultados. La prevalencia global del VPH en 194 estudiantes fue de 14.4\%. Las mujeres con dos o más parejas sexuales durante el último año presentaron mayor riesgo de infección por elVPH (RM 6.0 IC 1.7-21.1), al igual que las que utilizaron anticonceptivos hormonales y espermicidas en su última relación sexual (RM 3.0 IC 1.0-8.7). Los hombres que consumieron cocaína tuvieron más riesgo de infección por el VPH (RM 7.6 IC 1.3-45.1). Conclusiones La prevalencia del VPH es relativamente alta. La utilización del VPH como un marcador biológico de comportamientos sexuales en mujeres es pertinente; en hombres, es necesario ampliar la muestra. El texto completo en inglés de este artículo está disponible en:http:/ /www.insp.mx/salud/index.html
\end{abstract}

Palabras clave: papilomavirus humano; jóvenes universitarios; marcadores bioló gicos; México

\author{
Sánchez-Alemán MA, Uribe-Salas F, \\ Conde-González $\mathrm{CJ}$. \\ Human papillomavirus infection is a possible \\ biological marker of sexual behavior \\ among university students. \\ Salud Publica Mex 2002;44:442-447. \\ The English version of this paper \\ is available at: http://www.insp.mx/salud/index.html
}

\begin{abstract}
Objective. To estimate the prevalence of Human papillo mavirus (HPV) among university students and to use it as a biological marker to assess sexual behavior. Material and Methods. A cross-sectional study was carried out between 2000 and 2001 among 194 students at U niversidad Autónoma del Estado de Morelos, Mexico.A data collection instrument was applied and genital samples were taken to detect oncogenic H PV D N A. D ata were analyzed using the chi-squared test and odds ratios. Results 0 verall HPV prevalence was $14.4 \%$. W omen who had had two or more sexual partners during the previous year showed a greater risk of HPV infection (OR 6.0,95\% CI 1.7-21.1), as did women who had used oral contraceptives and spermicides at their latest intercourse (O R 3.0, 95\% Cl 1.0-8.7). Males who consumed cocaine were at a greater risk of HPV infection (OR 7.6, 95\% Cl 1.3-45.1). Conclusions HPV prevalence is relatively high. HPV is a reliable biological marker of sexual behavior among females.A greater sample size may be needed to assess its reliability among men. The English version of this paper is available at: http:// www.insp.mx/salud/index.html
\end{abstract}

Key words: human papillomavirus; university students; biological markers; Mexico

Miguel A ngel Sánchez-A lemán agradece al Consejo N acional de Ciencia yTecnología por la beca recibida, con lo cual se sustentó parte del presente trabajo.

(1) Centro de Investigaciones sobre Enfermedades Infecciosas, Instituto N acional de Salud Pública. Cuernavaca, Morelos, México.

Fecha de recibido: 18 de febrero de 2002 - Fecha de aprobado: 25 de junio de 2002

Solicitud de sobretiros: Dr. Carlos J C onde-G onzález. C entro de Investigaciones sobre Enfermedades Infecciosas. Instituto N acional de Salud Pública. Avenida Universidad 655, Colonia Santa María A huacatitlán, 62508 Cuernavaca, Morelos, México.

Correo electrónico: cjconde@ correo.insp.mx 
L presencia de anticuerpos contra el virus del herpes simple tipo 2 (VHS-2) ha sido considerada como el marcador biológico óptimo para estudiar el comportamiento sexual en poblaciones humanas, ya que relaciona los comportamientos sexuales con la existencia de anticuerpos contra el virus. ${ }^{1,2}$ Sin embargo, el desarrollo de métodos moleculares para diagnosticar diferentes infecciones de transmisión sexual (ITS) presentes en el momento, ha posibilitado la utilización de otros agentes como marcadores biológicos, potencialmente útiles para evaluar en estudios epidemiológicos el comportamiento sexual.

La ITS producida por el virus del papiloma humano (VPH) presenta dos características importantes. En primer lugar, puede identificarse mediante técnicas moleculares, como la reacción en cadena de la polimerasa ( $\mathrm{RCP})$ y la captura de híbridos $(\mathrm{CH}){ }^{3}$ Segundo, la papilomatosis tiene una prevalencia alta de acuerdo con estimaciones hechas por la Organización Mundial de la Salud, al reconocer que en 1995 se presentaron 30 millones de casos nuevos en el mundo. ${ }^{4}$ Esta alta frecuencia se debe en parte a que dicho virus se compone de más de 85 genotipos, de los cuales 30 son transmitidos por contacto sexual y de éstos, al menos 13 se han asociado con el desarrollo de cáncer cervicouterino. ${ }^{5}$

Los estudiantes universitarios son un grupo compuesto en su mayoría por jóvenes de 18 a 25 años de edad y es generalmente en esta etapa de la vida cuando inician sus relaciones sexuales ${ }^{6}$ y comienzan a exponerse a las ITS, como la originada por el VPH. El presente trabajo tiene como objetivos estimar la prevalencia de infección por el VPH en estudiantes universitarios, y utilizar dicha frecuencia como un marcador biológico para evaluar el comportamiento sexual en este grupo poblacional de riesgo desconocido.

\section{Material y métodos}

Se realizó un estudio transversal con un muestreo por conveniencia en alumnos de uno $u$ otro sexo de las facultades de medicina, farmacia, derecho, educación, administración, psicología y biología, de la Universidad Autónoma del estado de Morelos (UAEM), durante el periodo de noviembre de 2000 a mayo de 2001. Previo consentimiento informado, los estudiantes proporcionaron muestras genitales y contestaron una encuesta autoaplicada sobre características sociodemográficas y de comportamiento sexual. Las muestras genitales se tomaron con el "Specimen Collection Kit" [Digene, Maryland, Estados Unidos de América (EUA)]. La muestra de las mujeres se obtuvo por autotoma de células vaginales, y para ello cada participante recibió instrucción verbal de un miembro del equipo de investigación para que, mediante un hisopo estéril, tomaran una muestra del introito vaginal por medio de un frotamiento vigoroso de las paredes. En los hombres un miembro del equipo de investigación previamente entrenado colectó células exfoliativas del surco balano-prepucial y el glande, utilizando un hisopo estéril para hacer un frotamiento vigoroso del área mencionada. En ambos casos, el hisopo con el material colectado se depositó en un tubo con medio de mantenimiento que, de inmediato, se transportó al laboratorio y fue congelado a $-20{ }^{\circ} \mathrm{C}$ hasta su procesamiento.

La identificación de la infección por los VPH oncogénicos o de alto riesgo se hizo mediante la técnica de $\mathrm{CH}$ de acuerdo con las instrucciones del fabricante (Digene HPV test Hybrid Capture II), la cual busca ADN del VPH. Este procedimiento desnaturaliza el ADN de la muestra para hibridarlo con una mezcla de sondas de ARN de 13 diferentes VPH oncogéni$\cos (16,18,31,33,35,39,45,51,52,56,58,59$ y 68). El híbrido (ADN:ARN) se retiene mediante un anticuerpo a una microplaca de 96 pozos; posteriormente se adicionan anticuerpos IgG antihíbridos conjugados a fosfatasa alcalina; se agrega el substrato y se detecta la reacción mediante quimioluminiscencia, cuantificada ésta en un luminómetro al compararla con los controles respectivos. El análisis estadístico exploratorio, univariente, bivariante y estratificado se realizó con el paquete SPSS 10.0; se emplearon pruebas de $\chi^{2}$ y razones de momios $(R M)$, estas últimas con intervalos de confianza (IC 95\%).

\section{Resultados}

Participaron en el estudio 233 alumnos, de los cuales 39 no habían iniciado su vida sexual (30 mujeres y nueve hombres). En ninguno de estos últimos se detectó ADN del VPH. Para los análisis posteriores se consideró sólo a los individuos que habían iniciado su vida sexual al momento de la encuesta, 71 hombres y 123 mujeres. La prevalencia del VPH en esta población fue de $14.4 \%$; la frecuencia de la infección fue mayor en mujeres que en hombres: 17.9 vs $8.5 \%$, respectivamente $(R M=2.4$; IC 95\% 0.9-6.1).

Las relaciones sexuales con parejas ocasionales mostraron un comportamiento contrastante, pues aumentó la prevalencia del VPH en los hombres y disminuyó para las mujeres. Las relaciones con parejas del mismo sexo se asociaron con un incremento en la presencia del virus en los varones. No se encontró significancia estadística en ninguna de las variables anteriores (cuadro I). 
En las mujeres se detectaron tres asociaciones significativas: haber tenido dos o más parejas sexuales en toda la vida, haber tenido dos o más parejas sexuales durante el último año, y haber utilizado en la última relación sexual anticonceptivos hormonales o espermicidas. En hombres sólo el consumo de cocaína se asoció de manera significativa con la presencia del VPH (cuadro I).

\section{Cuadro I}

Características sociodemográficas y de comportamiento sexual en estudiantes de la Universidad Autónoma del estado de Morelos en función de la prevalencia del VPH. Morelos, MÉxico, 2000-2001

\begin{tabular}{|c|c|c|c|c|c|c|c|c|}
\hline \multirow[b]{2}{*}{ Características } & \multicolumn{4}{|c|}{ Mujeres } & \multicolumn{4}{|c|}{ Hombres } \\
\hline & $\mathrm{n}^{*}$ & Prev & RM & IC $95 \%$ & $\overline{n^{*}}$ & Prev & RM & IC $95 \%$ \\
\hline \multicolumn{9}{|l|}{ Edad } \\
\hline $16-20$ años & 46 & 13.0 & 2.1 & $0.2-19.0$ & 22 & 9.1 & 1.1 & $0.1-1.5$ \\
\hline $21-25$ años & 62 & 24.2 & 4.5 & $0.5-36.9$ & 37 & 8.1 & 1.0 & $0.1-10.3$ \\
\hline 26 y más años & 15 & 6.7 & & & 12 & 8.3 & & \\
\hline \multicolumn{9}{|l|}{$\mathrm{N}$ ivel socioeconómico } \\
\hline Alto & 70 & 18.6 & 1.2 & $0.5-3.2$ & 37 & 10.8 & 1.9 & $0.3-11.3$ \\
\hline Bajo/medio & 51 & 15.7 & & & 34 & 5.9 & & \\
\hline \multicolumn{9}{|l|}{ Alcohol } \\
\hline Cada semana /cada mes & 91 & 22.0 & 3.9 & $0.9-18.0$ & 56 & 8.9 & 1.0 & $0.1-9.3$ \\
\hline N unca / casi nunca & 30 & 6.7 & & & 11 & 9.1 & & \\
\hline \multicolumn{9}{|l|}{ Cocaína } \\
\hline Sí & 3 & 0.0 & - & - & 10 & 30.0 & 7.6 & $1.3-45.1^{\ddagger}$ \\
\hline No & 115 & 18.3 & & & 56 & 5.4 & & \\
\hline
\end{tabular}

Antecedentes de ITS

\begin{tabular}{lrrrrrrrr} 
Sí & 5 & 40.0 & 3.2 & $0.5-20.6$ & 3 & 0.0 & - & - \\
\hline No / no sé & 117 & 17.1 & & & 68 & 8.8 & & \\
$\begin{array}{l}\text { Parejas en toda la vida } \\
\text { 2 y más }\end{array}$ & & & & & & & & \\
\hline 1 & 61 & 26.2 & 3.3 & $1.2-9.0^{\ddagger}$ & 56 & 8.9 & 1.2 & $0.1-11.0$ \\
\hline 1 & 61 & 9.8 & & & 13 & 7.7 &
\end{tabular}

Parejas durante el último año

\begin{tabular}{lcccccccc}
$\begin{array}{c}\text { Parejas durante el último ano } \\
2 \text { y más }\end{array}$ & 12 & 50.0 & 6.0 & $1.7-21.1^{\ddagger}$ & 25 & 8.0 & 0.8 & $0.1-5.0$ \\
\hline y y 1 & 105 & 14.3 & & & 43 & 9.3 &
\end{tabular}

Parejas en un mismo mes

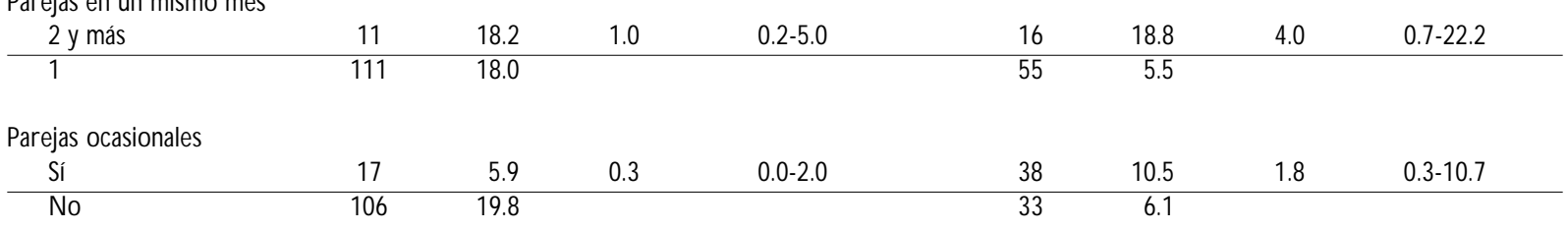

Parejas del mismo sexo

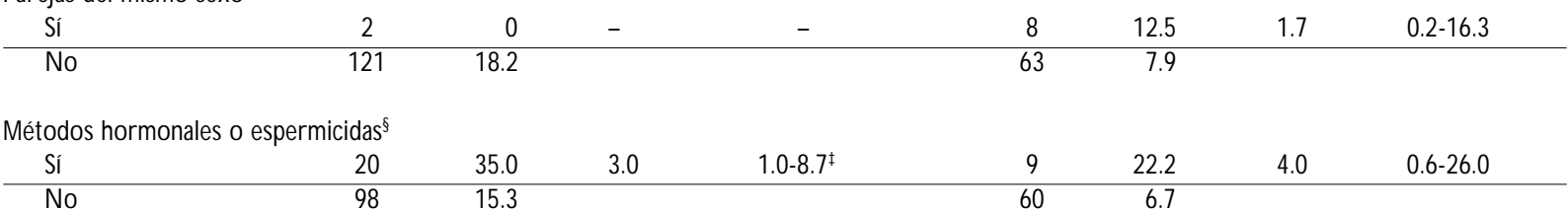

* Los individuos que faltan en cada estrato no contestaron la pregunta respectiva

‡ Inter valos de confianza significativos

$\S$ Pastillas, óvulos, geles, espumas, inyecciones o píldora de emergencia como anticonceptivos en la última relación sexual 
El uso de cocaína en varones estuvo asociado con la frecuencia en el consumo de alcohol, el número de parejas sexuales en diferentes periodos de tiempo y el haber tenido relaciones sexuales con parejas ocasionales (cuadro II).

\section{Discusión}

La prevalencia global del VPH (14.4\%) es alta en comparación con la frecuencia de anticuerpos contra el

\section{Cuadro II \\ USO DE COCAÍNA Y DIVERSAS CARACTERÍSTICAS SOCIODEMOGRÁFICAS Y DE COMPORTAMIENTO SEXUAL EN HOMBRes de LA UnIVERSIDd AUtónoma deL ESTAdo de Morelos. Morelos, México, 2000-2001}

Características

Nivel socioeconómico

\begin{tabular}{lrrr} 
Alto & 36 & $19.4 \%$ & 0.260 \\
\hline Medio & 18 & $16.7 \%$ & \\
\hline Bajo & 12 & $0.0 \%$ &
\end{tabular}

Consumo de alcohol

\begin{tabular}{lrrr} 
Cada semana & 16 & $37.5 \%$ & $0.004^{\ddagger}$ \\
\hline Cada mes & 24 & $12.5 \%$ & \\
\hline N unca / casi nunca & 23 & $0.0 \%$ &
\end{tabular}

Parejas en toda la vida

\begin{tabular}{lrrr}
6 y más & 18 & $38.9 \%$ & $0.005^{\ddagger}$ \\
\hline $3-5$ & 22 & $9.1 \%$ & \\
\hline 1 y 2 & 25 & $4.0 \%$ &
\end{tabular}

Parejas durante el último año

\begin{tabular}{lrrr}
3 y más & 10 & $50.0 \%$ & $0.005^{\ddagger}$ \\
\hline 2 & 14 & $14.3 \%$ & \\
\hline 0 y 1 & 38 & $7.9 \%$ &
\end{tabular}

Parejas en un mismo mes

\begin{tabular}{lrrr}
2 y más & 16 & $37.5 \%$ & $0.010^{\ddagger}$ \\
\hline 1 & 50 & $8.0 \%$ &
\end{tabular}

Parejas ocasionales

\begin{tabular}{lrrr} 
Sí & 37 & $27.0 \%$ & $0.002^{\ddagger}$ \\
\hline No & 29 & $0.0 \%$ &
\end{tabular}

Métodos hormonales o espermicidas ${ }^{\S}$

\begin{tabular}{lrrr} 
Sí & 9 & $33.3 \%$ & 0.140 \\
\hline No & 55 & $12.7 \%$ &
\end{tabular}

* Los individuos que faltan en cada estrato no contestaron la pregunta respectiva

₹ Diferencias estadísticamente significativas (95\%)

$\S$ Pastillas, óvulos, geles, espumas, inyecciones o píldora de emergencia como anticonceptivos en la última relación sexual
VHS-2 (5.9\%) reportada en estudiantes del mismo universo poblacional. ${ }^{7}$ Estos resultados son contrarios a lo esperado, si se consideran otros grupos poblacionales, como trabajadoras sexuales de la Ciudad de México (43\% VPH vs 65.1\% VHS-2) ${ }^{8,9}$ y mujeres mexicanas de muestras poblacionales (14.5\% VPH vs $29.8 \%$ VHS-2); ${ }^{10,11}$ en ambos casos la seroprevalencia del VHS2 es mayor que la prevalencia del VPH. La frecuencia del VPH en la muestra de universitarios podría ser aún mayor, debido a que sólo se identificaron 13 de los 30 genotipos virales que infectan el tracto genital y a que no se corroboró la calidad de las muestras biológicas a través de la determinación en ellas del gen de $\beta$-globina. No obstante, este estudio muestra que aun evaluando solamente los tipos oncogénicos del VPH, su frecuencia es alta en población joven y por ello se plantea utilizarla como un marcador biológico para evaluar el comportamiento sexual.

La autotoma de la muestra vaginal tiene la ventaja de una mayor aceptación, toda vez que la maniobra de una exploración ginecológica no era factible de practicarse en la población de estudio, además de que con ello se disminuiría la participación. Por otra parte, la autotoma vaginal tiene la desventaja de una menor sensibilidad y especificidad que la muestra cervical colectada por personal capacitado, sin embargo, un par de investigaciones demostraron una concordancia por arriba de $80 \%$ entre la autotoma vaginal y la colecta cervical. ${ }^{12,13}$

La prevalencia por el VPH fue mayor en mujeres que en hombres, y aunque dicha diferencia no fue significativa se decidió analizar por separado, en hombres y en mujeres, la frecuencia de infección por el VPH y su relación con los comportamientos sexuales y otras características sociodemográficas.

El 17.9\% del VPH que se encontró en la muestra de mujeres de la UAEM es mayor al reportado en mujeres de una muestra poblacional del estado de Morelos $(14.5 \%)^{10}$ y menor al encontrado en grupos con prácticas de riesgo ( $43 \%$ en trabajadoras sexuales y $69 \%$ en mujeres VIH positivas), ${ }^{8,14}$ estos datos sugieren que las mujeres de nuestro estudio probablemente se encuentren en una situación intermedia entre las poblaciones con prácticas de alto riesgo y los grupos con comportamientos de bajo riesgo.

En mujeres, el riesgo de infección por el VPH se asoció con el aumento en el número de parejas sexuales en diferentes periodos de tiempo. Este es un indicador de comportamiento sexual que nos habla del incremento de la probabilidad de entrar en contacto con una pareja sexual que porte al agente infeccioso. ${ }^{15}$ El número de parejas sexuales es un indicador de riesgo para adquirir ITS, que ha sido referido en forma 
constante en diversos estudios. ${ }^{16,17}$ Por otra parte, en mujeres, la utilización de anticonceptivos hormonales o espermicidas en la última relación sexual se asoció con la presencia del VPH; dicha relación ha sido reportada en universitarias de Canadá ${ }^{18}$ y EUA. ${ }^{19}$ Así, el uso de métodos anticonceptivos, hormonales o espermicidas, podría ser un reflejo de la no utilización del condón.

Si bien el estudio del VPH se ha enfocado a las mujeres, en nuestra investigación también se incluyeron muestras de 71 varones sexualmente activos, con una prevalencia del VPH de alto riesgo de $8.5 \%$. En Morelos un estudio en 96 hombres encontró una frecuencia de $19.8 \%$ de VPH oncogénicos; ${ }^{20}$ en esa investigación se empleó como técnica de detección la RCP y se confirmó la calidad de las muestras. Con los universitarios de la UAEM se utilizó la técnica de $\mathrm{CH}$ y no se verificó la calidad del material colectado; estas son parte de las posibles causas de la diferencia entre ambos estudios, además de la diversidad entre las poblaciones.

En hombres, la infección por el VPH se asoció con el consumo de cocaína; el uso de esta droga se ha relacionado con otras ITS en diversos estudios. ${ }^{21,22} \mathrm{La}$ cocaína por sí misma no tiene efecto sobre la prevalencia del VPH, sin embargo, su utilización aumentó de manera directamente proporcional con respecto a algunos comportamientos sexuales de riesgo para adquirir ITS en los universitarios estudiados; esta misma relación la reportó Hser al asociar el consumo de cocaína con el haber tenido más de una pareja sexual durante el último año, y la no utilización de condón en la última relación sexual. ${ }^{23}$

Algunas limitaciones de nuestra investigación fueron la autoselección de la población y el tamaño de la muestra estudiada. Esta última originó una baja potencia estadística en el cálculo de los riesgos de infección por el VPH, lo que daría como resultado la ausencia de diferencias significativas cuando en realidad sí existen. La baja potencia se pone de manifiesto particularmente en los hombres, los cuales por cuestiones culturales no se encuentran habituados a cooperar cuando se trata de proporcionar muestras biológicas tomadas directamente de genitales.

La prevalencia del VPH es alta en la población universitaria estudiada; en mujeres existe una asociación entre la infección por el VPH y comportamientos sexuales de riesgo para adquirir ITS, por lo cual se pone de manifiesto la pertinencia de la utilización del VPH como un marcador biológico de comportamiento sexual en mujeres. En hombres es necesario ampliar la muestra para obtener conclusiones, ante su limitada participación en esta investigación.

\section{Agradecimientos}

Los autores agradecen a las autoridades de las diferentes facultades de la UAEM por todo el apoyo recibido; a la M en CS, Cecilia Gayet, por la revisión de la encuesta, y a la TLC, Pilar Hernández, por la asesoría técnica para las pruebas de $\mathrm{CH}$.

\section{Referencias}

1.N ahmias AJ, Lee FK, Beckman-N ahmias S. Seroepidemiological and sociological patterns of herpes simplex virus infection in the world. Scan J Infect D is Suppl 1990;69:19-36.

2. Cowan FC, Johnson AM, A shley R, Corey L, Mindel A. Antibody to herpes simplex virus type 2 as serological marker of sexual lifestyle in populations. BMJ 1994;309:1325-1329.

3. Peyton CL, Schiffman M, Lörinckz AT, Hunt W C , Mielzynska I, Bratti C et al. Comparision of PCR and hybrid capture-based human papillomavirus detection systems using multiple cervical specimen collection strategies.J Clin Microbiol 1998;36:3248-3254.

4. World Health 0 rganization. Sexually transmitted disease. Three hundred and thirty three million new curable cases in 1995. Ginebra, 1995. 5. Zur-Hausen H. Papillomaviruses in human cancers. Proc Assoc Am Physicians 1999;111:581-587.

6. Ibáñez-Brambila B, 0 drizola-U rbina A. Religiosidad y conducta sexual premarital en estudiantes universitarios. Enseñanza e investigación en psicología 1997;2:124-132.

7. Sánchez-A lemán MA. Prevalencia y factores asociados a la infección por Treponema pallidum, Virus del Herpes Simple tipo 2 y Virus del Papiloma Humano en estudiantes universitarios (tesis de Maestría). Cuernavaca: Escuela de Salud Pública de México/ Instituto N acional de Salud Pública, 2001.

8. Juárez-Figueroa LA, W heeler CM, U ribe-Salas FJ, Conde-González CJ, Zamilpa-Mejía LG , G arcía-C isneros S. Human Papillomavirus, a highly prevalent sexually transmitted disease among female sex workers from Mexico City. Sex Transm D is 2001;28:125-130.

9. U ribe-Salas F, Hernández-Avila M, Juárez-Figueroa L, C onde-González CJ, U ribe-Zúñiga P. Risk factors for herpes simplex virus type 2 infection among female commercial sex workers in Mexico City. Int J STD AIDS $1999 ; 10: 105-111$.

10. Lazcano-Ponce E, Herrero R, Muñoz N , Cruz A, Shah KV, Alonso P et al. Epidemiology of HPV infection among Mexican women with normal cervical cytology. Int J Cancer 2001;91:412-420.

11. Lazcano-Ponce E,Smith J, Muñoz N , Conde-G onzález C J, Juárez-Figueroa $\mathrm{L}, \mathrm{C}$ ruz $\mathrm{A}$ et al. High prevalence of antibodies to herpes simplex virus type 2 among middle-aged women in Mexico City, Mexico. Sex Transm Dis 2001;28:270-276.

12.W right TC, D enny L, Kuhn L, Pollack A, Lörinckz AT. H PV D N A testing of self-collected vaginal samples compared with cytologic screening to detect cervical cancer. JAMA, 2000;283:81-86.

13. G ravitt PE, Lacey JV, Brinton LA, Barnes W A, Kornegay JR, G reenberg $M D, G$ reene $S M$ et al. A. Evaluation of self-collected cervicovaginal cell samples for human papillomavirus testing by polymerase chain reaction. Cancer Epidemiol Biomarkers Prev 2001;10:95-100.

14.Volkow P, Rubí S, Lizano M, C arrillo A,Vilar-Compte D, G arcía-Carran$\mathrm{ca} A$ et al. High prevalence of oncogenic human papillomavirus in the genital tract of women with human immunodeficiency virus. G ynecol 0 ncol 2001;82:27-31. 
15. SpiraA, Bajos N ,ACSF Group staff. Sexual behavior and AID S. A shgate Publishing Company, 1994.

16. Ho GY, Bierman R, Beardsley L, Chang CJ, Burk RD. N atural history of cervicovaginal papillomavirus infection in young women. $\mathrm{N}$ Engl J Med 1998:338:423-428

17. Sellors JW, Mahony JB, Kaczorowski J, Lytwyn A, Bangura H, C hong $S$ et al. Prevalence and predictors of human papillomavirus infection in women in $\mathrm{O}$ ntario, Canada. Survey of HPV in $\mathrm{O}$ ntario W omen (SHOW ) Group, CMAJ, 2000; 163:503-508

18. Richardson H, Franco E, Pintos J, Bergeron J,A rella M, Tellier P. D eterminants of low-risk and high-risk cervical human papillo mavirus infections in Montreal university students. Sex Transm D is 2000;27:79-86.

19. Burk RD, Ho GY, Beardsley L, Lempa M, Peters M, Bierman R. Sexual behavior and partner characteristics are the predominant risk factors for genital human papillomavirus infection in young women. J Infect $D$ is 1996;174:679-689.

20. Lazcano-Ponce E, Herrero R, Muñoz N , Hernández-Avila M, Salmerón J, LeyvaA et al. High prevalence of human papilomavirus infection in Mexican males. Sex Transm D is 2001;28:277-280.

21. Fleming DT, McQ uillan GM, Johnson RE, N ahmias AJ, A ral SO, Lee FK et al. Herpes Simplex Virus type 2 in the United States, 1976 to 1994. N Engl J Med 1997;337:1105-1111.

22. Poulin C, Michel A, Bernier F, Ringuet J, Joly JR. Prevalence of Chlamydia trachomatis, Neisseria gonorrhoeae, and HIV infection among drug users attending an STD/HIV prevention and needle-exchange program in Q uebec City, C anada. Sex Transm D is 1999;26:410-420.

23. Hser Y, Chou ChP, H offman V,Anglin D. C ocaine use and high-risk sexual behavior among STD clinic patients. Sex Transm D is 1999;26: 82-86. 